\title{
Optimizing Vehicle Motion Control for Generating Multiple Sensations
}

\author{
Moad Kissai ${ }^{1}$, Xavier Mouton $^{2}$, Bruno Monsuez ${ }^{1}$, Didier Martinez ${ }^{2}$, and Adriana Tapus ${ }^{1}$
}

\begin{abstract}
Most of automotive researches focus on autonomous vehicles. Studies regarding trajectory planning and trajectory tracking became preponderant. As in case of commercial ground vehicles there is a driver in the loop, one should raise the important question of how the trajectory should be tracked. In this paper, we investigate the influence of controlling integrated chassis systems on the vehicle's behavior. A fixed Model Predictive Control is used to track the trajectory. Tunable vehicle motion control is however used to provide different motion feelings. Results show that a specific trajectory could be followed in different manners. Therefore, vehicle dynamics can be and should be controlled in such a way to generate adaptive trust feelings to passengers in case of autonomous driving.
\end{abstract}

Index Terms-Autonomous Vehicles, Vehicle Motion Control, Chassis Systems, Control Allocation, Optimization, Cosimulation.

\section{INTRODUCTION}

Advanced Driver Assistance Systems (ADAS) are one of the most important features in ground vehicles. Normal drivers are usually trained to handle a vehicle in normal situations. In hazardous situations, vehicle dynamics become less obvious to predict. Assistance systems provide then the necessary control to help drivers continue their maneuvers with less damages. Both car manufacturers and equipment suppliers are actively working on developing new technologies to enhance the vehicle's safety. It started with the Anti-lock Braking System (ABS) in 1978 [1]. This system consists of relaxing the brakes to regain adherence and then braking again to decelerate the vehicle. The major goal of ABS was to reduce the distance of braking for better longitudinal control. A significant progress was provided by the Electronic Stability Program (ESP) in 1992 [1]. This system consists of a differential braking between right and left tires to provide a yaw moment and control the yaw rate. This is particularly beneficial for obstacle avoidance for example. The ESP brought then a lateral control to help drivers stabilize their vehicles. A natural evolution would be a global control or fully autonomous vehicles. In this case, longitudinal, lateral, and eventually vertical control logics should be coordinated to handle vehicle and tire dynamic couplings [2]. Moreover, with the addition of new technologies, vehicles become over-actuated [3]. Control

\footnotetext{
${ }^{1}$ Moad Kissai, Bruno Monsuez, and Adriana Tapus are with ENSTA ParisTech, Autonomous Systems and Robotics Lab, Department of Computer and System Engineering, 828 Boulevard des Marchaux, 91762 Palaiseau Cedex, France moad.kissai, bruno.monsuez, adriana.tapus\}ensta-paristech.fr

${ }^{2}$ Xavier Mouton, and Didier Martinez are with Group Renault, Chassis Systems Department, 1 Avenue du Golf, 78280 Guyancourt, France \{xavier.mouton, didier.d.martinez\}erenault.com
}

should be allocated to the right actuators and effectors no matter what the situation is.

In this context, different strategies have been adopted in the literature. Two major control approaches have been distinguished in [2]: the Downstream Approach and the Upstream Approach. In the first approach, data-based and techniques such as Artificial Neural Network [4], or rulebased techniques such as Fuzzy Logic Control [5] are used downstream systems' standalone controllers. These techniques handle specific use-cases and depend on designers' expertise. As no customer feedback about autonomous vehicles is available, coordination should rather be more general independent from any use-case. This can only be done by describing mathematically the dynamic couplings and use optimization-based techniques. A sufficient amount of information is then required to distribute the commands and allocation is done upstream the systems' standalone controllers [2]. Optimization-based control allocation techniques offer additional attractive features. In fact, optimization is carried out by taking into account multi-objectives. In [6],[7],[8] for example, energy consumption has been used as a criterion to allocation precision and stability. This is particularly beneficial for electric vehicles [9]. Having multiple ADAS or chassis systems can then offer supplementary possibilities. Vehicle motion can be then modified in a way to fulfill multiple objectives.

For autonomous vehicles however, qualitative objectives could be required. When a driver has its hands off the steering wheel, if an unexpected motion is generated, he could be tempted to regain control of the vehicle. In case of a vehicle equipped with an Electric Power-Assisted Steering (EPAS) system, this may present few risks for drivers' hands due to the important amount of steering wheel torque [3]. One way to prevent this, is to tune the vehicle motion in a way to generate expected motions like a human being would do. To the best of our knowledge, there exist two method to tune the vehicle behavior. Either we act on motion references to be followed by the vehicle so the controllers impose different commands to actuators, or we modify the control allocation strategy so the commands are distributed differently.

The purpose of this paper is to compare both approaches to highlight the advantages and drawbacks of each one. The goal is to give few insights about the ability of modern control techniques to provide additional degrees of freedom regarding motion feelings control. Investigations aiming to adapt these techniques to drivers' profiles are still needed with the help of experiments. Here, a Model Predictive Control (MPC) provided by LMS Imagine.Lab AMESim ${ }^{\circledR}$ 
is used for trajectory tracking. Focus has been put more on vehicle motion control tuning. A Gain-Scheduled $\mathcal{H}_{\infty}$ controller has been selected as the high-level controller for its robustness and dynamic couplings management. Optimizationbased Control Allocation methods are then used to distribute the commands into the different actuators taking into account tire potential. Results by co-simulation of AMESim ${ }^{\circledR}$ and Matlab/Simulink ${ }^{\circledR}$ showed attractive improvements brought by these control methods to handle on one hand complex coupled situations, and on the other hand, to provide different motions while tracking the same trajectory.

The rest of the paper is structured as follows: We start in Section II by presenting the different modelings required for the problem definition. In Section III, the different control methods adopted are described. The two approaches enabling the motion behavior tuning are both presented in Section IV. Section V presents results obtained by co-simulation of Matlab/Simulink ${ }^{\circledR}$ and AMESim $^{\circledR}$. A discussion about future cars challenges and the relevance of this work is provided in Section VI. Conclusions and future works are outlined in Section VII.

\section{SYSTEM MODELING}

The vehicle considered in this paper is equipped with an Electric Power-Assisted Steering (EPAS) system, an Electronic Stability Program (ESP), and a Torque Vectoring (TV) system. This latter is realized by means of four motor-wheels. The EPAS is mainly controlled by the MPC. The goal is to study interactions between systems and what a better coordination can bring to the vehicle motion control.

As no active suspensions are considered, a planar vehicle model can be adopted. Nevertheless, as the ESP and TV are based on differential forces between right and left wheels, a four-wheeled vehicle model is necessary [10]. Using Newton's laws of motion, one can find easily the following staterepresentation [3]:

$$
\left\{\begin{aligned}
& {\left[\begin{array}{c}
\dot{V}_{x} \\
\ddot{\psi}
\end{array}\right] }=\left[\begin{array}{cc}
0 & V_{y} \\
0 & 0
\end{array}\right]\left[\begin{array}{c}
V_{x} \\
\dot{\psi}
\end{array}\right] \\
&+ {\left[\begin{array}{cc}
\frac{1}{M} & 0 \\
0 & \frac{1}{I_{z z}}
\end{array}\right]\left[\begin{array}{l}
F_{x_{t o t}} \\
M_{z_{t o t}}
\end{array}\right] } \\
& {\left[\begin{array}{c}
V_{x} \\
\dot{\psi}
\end{array}\right]=\left[\begin{array}{ll}
1 & 0 \\
0 & 1
\end{array}\right]\left[\begin{array}{c}
V_{x} \\
\dot{\psi}
\end{array}\right] }
\end{aligned}\right.
$$

With:

- $V_{x}$ : longitudinal velocity of the vehicle,

- $V_{y}$ : lateral velocity of the vehicle,

- $\dot{\psi} \quad$ : yaw rate of the vehicle,

- $F_{x_{t o t}}$ : sum of longitudinal forces applied at the vehicle's Center of Gravity (CoG),

- $M_{z_{\text {tot }}}$ : sum of yaw moments applied at the vehicle's CoG,

- $M$ : vehicle's overall mass,

- $I_{z z}$ : vehicle's yaw moment of inertia.
Where:

$$
\left\{\begin{aligned}
F_{x_{t o t}}= & \left(F_{x_{f l}}+F_{x_{f r}}\right) \cos \left(\delta_{f}\right)+F_{x_{r l}}+F_{x_{r r}} \\
M_{z_{t o t}}= & \left(F_{x_{f l}}+F_{x_{f r}}\right) l_{f} \sin \left(\delta_{f}\right) \\
& +\left(F_{x_{f r}}-F_{x_{f l}}\right) \frac{t_{r}}{2} \cos \left(\delta_{f}\right) \\
& +\left(F_{x_{r r}}-F_{x_{r l}}\right) \frac{t_{r}}{2}
\end{aligned}\right.
$$

With:

- $F_{x_{f, r}}$ : front-right longitudinal force,

- $F_{x_{f, l}}$ : front-left longitudinal force,

- $F_{x_{r, r}}$ : rear-right longitudinal force,

- $F_{x_{r, l}}$ : rear-left longitudinal force,

- $\delta_{f} \quad$ : front steering angle,

- $l_{f} \quad$ : distance between the front axle and the vehicle's $\mathrm{CoG}$,

- $t_{r} \quad$ : vehicle's track.

Note that we have considered only controllable forces to distribute by the control allocation algorithm. In fact, the lateral force induced by the front steering is controlled by the MPC. Introducing this force also in the vehicle motion control induces request conflicts ${ }^{1}$.

Regarding tire forces, a linear tire model with varying parameters developed in [11] is used to take into account the couplings at the tire level. The forces are expressed as following:

$$
\left\{\begin{array}{l}
F_{x}=C_{s}^{*}\left(\alpha, \mu, F_{z}\right) \kappa \\
F_{y}=C_{\alpha}^{*}\left(\kappa, \mu, F_{z}\right) \alpha
\end{array}\right.
$$

where:

- $\kappa \quad:$ the longitudinal slip,

- $\alpha \quad$ : the side-slip,

- $\mu \quad:$ the friction coefficient,

- $F_{z} \quad:$ the vertical load,

- $C_{s}^{*}\left(\alpha, \mu, F_{z}\right)$ : the tire varying longitudinal stiffness with respect to the side-slip $\alpha, \mu$, and $F_{z}$,

- $C_{\alpha}^{*}\left(\kappa, \mu, F_{z}\right)$ : the tire varying cornering stiffness with respect to the longitudinal slip $\kappa, \mu$, and $F_{z}$.

Detailed expressions of $C_{s}^{*}\left(\alpha, \mu, F_{z}\right)$ and $C_{\alpha}^{*}\left(\kappa, \mu, F_{z}\right)$ can be found in [11].

In order to respect the friction ellipse concept [12], dynamic constraints are added [11]:

$$
\left\{\begin{array}{l}
F_{x} \leq \sqrt{\left(\mu F_{z}\right)^{2}-F_{y}^{2}} \\
F_{y} \leq \sqrt{\left(\mu F_{z}\right)^{2}-F_{x}^{2}}
\end{array}\right.
$$

\section{VEHICLE MOTION CONTROL}

Today's vehicles are over-actuated [3]. Integrated systems are expected to grow in number in the upcoming years due to the road-map towards autonomous vehicles. A discussion about control architectures to be adopted in order to face the growing number of integrated systems is provided in [2]. A multi-layered architecture is claimed to present several advantages, especially when systems coordination is located

\footnotetext{
${ }^{1}$ This case has been verified by simulation
} 
upstream the systems themselves. The same approach is adopted here. The control architecture is divided in a highlevel control to generate the required sum of forces and moments necessary to move the vehicle, a middle-level control is used to distribute optimally the commands into the four wheels, and a low-level control is used to transform the forces into torques to be generated by the different actuators.

\section{A. High-Level Control}

The objective of this layer is to generate the generalized forces and moments to be applied at the vehicle's CoG. Equations (1) and (2) are considered. The model can be then considered as linear with the varying parameter $V_{y}$. To take into account this variation, a gain scheduled controller is chosen with $V_{y}$ as the scheduling variable. In fact, today's vehicles are equipped with an inertial measurement unit and a yaw rate sensor ${ }^{2}$. Therefore, $V_{y}$ can be estimated using a simplified vehicle model as in [3].

Moreover, as stated in [13], vehicle motion control faces several problems related to parameter uncertainties. The control should valid whether there is only the driver in the vehicle or with other passengers, whether tires are brand new or not and so on. A robust controller is then needed. The $\mathcal{H}_{\infty}$ synthesis has been selected to be able to optimize the controller parameters. As this synthesis usually generate high order controllers [14], a common practice consists on reducing the controller in the frequency domain while keeping an acceptable level of robustness [15]. Here, a different methodology is adopted. As most industrials prefer PID controllers for implementation issues, we add the controller structure as a requirement in the $\mathcal{H}_{\infty}$ synthesis. To choose the suitable structure to be adopted, a pre-study of the plant is required. We suppose that in stable maneuvers $V_{y}$ has a very low value. Therefore, a decoupled controller can be chosen. Moreover, due to the integral characteristic of each control axis, a PI controller can be chosen at each axis. Four tunable parameters are then chosen in the optimization $\mathcal{H}_{\infty}$ problem. More details about this methodology can be found in [16].

Regarding performance weighting functions, closed loop shaping is used for defining control design requirements as in [17]. We choose here a steady-state offset less than $1 \%$, a closed-loop bandwidth higher than $1 \mathrm{~Hz}^{3}$, and an amplification of high-frequency noise less than a factor 3 . The $\mathcal{H}_{\infty}$ synthesis is iterated for different values $V_{y}$. A gain-scheduled $\mathcal{H}_{\infty}$ controller is finally synthesized, which depends on $V_{y}$ estimations.

One major difference with respect to [17] investigations is the scheduling parameter. In our case, the gain-scheduling is only used for the high-level control. In [17] gain-scheduling is used to coordinate subsystems depending on a stability index. In this paper, optimization-based control allocation techniques are selected to handle subsystems coordination.

\footnotetext{
${ }^{2}$ According to the co-authors working in the Group Renault.

${ }^{3}$ To prevent the control allocation algorithm from operating at $100 \mathrm{~Hz}$ frequencies for example.
}

\section{B. Middle-Level Control}

The goal of middle-level control is to optimally distribute the generalized forces generated by the high-level control to ensure the execution of these forces. As long as an overactuated system is concerned, multiple objectives can be fulfilled. This is very important for this study as several problems are aimed to be addressed regarding autonomous vehicles. Optimization-based control allocation techniques are then selected to handle the forces distribution problem.

The control allocation problem is defined as follows [18]: find the control vector, $\vec{u} \in \mathbb{R}^{n}$ such that

$$
\mathbf{B}_{\text {eff }} \vec{u}=\vec{v}_{\text {des }}
$$

subject to

$$
\left\{\begin{aligned}
& \vec{u}_{\min } \leq \vec{u} \leq \vec{u}_{\max } \\
& \dot{\vec{u}} \leq \dot{\vec{u}}_{\max }
\end{aligned}\right.
$$

where $\mathbf{B}_{\text {eff }} \in \mathbb{R}^{m \times n}$ is a control effectiveness matrix, $\vec{u}_{\text {min }} \in \mathbb{R}^{n}$ and $\vec{u}_{\text {max }} \in \mathbb{R}^{n}$ are the lower and upper position limits, respectively, $\dot{\vec{u}} \in \mathbb{R}^{n}$ is the control rate, $\dot{\vec{u}}_{\max } \in \mathbb{R}^{n}$ is the maximum control rate, $\vec{v}_{\text {des }} \in \mathbb{R}^{m}$ are the desired accelerations, $n$ is the number of control effectors, and $m$ is the number of axes to control.

Several solvers can be used to solve a multi-objective problem. Sequential Least Squares (SLS) uses a two stage Active Set Algorithm (ASA) to handle two optimization problems [19]. The Weighted Least Squares (WLS) solves the bounded least squares problem using one stage ASA after few matrix transformations by means of different weights [19]. Other techniques not based on ASA that can be cited are Cascading Generalized Inverses (CGI) [20] and the FixedPoint Iteration (FPI) [21]. We choose here the WLS based on ASA for its flexibility to express multiple objectives, and for its relative rapidity due to its one stage formulation. The optimal solution is then:

$$
\vec{u}_{\text {opt }}=\arg \min _{\vec{u}_{\text {min }} \leq \vec{u} \leq \vec{u}_{\max }} \sum_{l} \gamma_{i}\left\|\mathbf{W}_{\mathbf{i}}\left(\mathbf{B}_{\mathbf{i}} \vec{u}-\vec{v}_{i}\right)\right\|^{2}
$$

- $\vec{u}_{\text {opt }} \quad$ : optimal control vector,

- $l \quad$ : number of objectives,

- $\gamma_{i} \quad$ : weight of the $i^{\text {th }}$ objective,

- $\mathbf{W}_{\mathbf{i}}$ : non-singular weighting matrices defining preferences of each axis,

- $\overrightarrow{v_{i}} \quad$ : desired vector of the $i^{\text {th }}$ objective,

- $\mathbf{B}_{\mathbf{i}}$ : effectiveness matrix relating the control vector to the desired $i^{\text {th }}$ objective.

Regarding the EPAS-ESP-TV coordination case:

$$
\vec{u}=\left[\begin{array}{c}
F_{x_{f l}} \\
F_{x_{f r}} \\
F_{x_{r l}} \\
F_{x_{r r}}
\end{array}\right]
$$

$\vec{u}_{\text {min }}$ and $\vec{u}_{\text {max }}$ reflect tire limits with respect to the friction ellipse concept (7) and (8). Regarding vertical forces, we 
have [3]:

$$
\left\{\begin{array}{l}
F_{z_{f l}}=\frac{1}{2} M g \frac{l_{r}}{L}-\frac{1}{2} M a_{x} \frac{h}{L}-\frac{1}{2} M a_{y} \frac{h}{t_{r}} \\
F_{z_{f r}}=\frac{1}{2} M g \frac{l_{r}}{L}-\frac{1}{2} M a_{x} \frac{h}{L}+\frac{1}{2} M a_{y} \frac{h}{t_{r}} \\
F_{z_{r l}}=\frac{1}{2} M g \frac{l_{f}}{L}+\frac{1}{2} M a_{x} \frac{h}{L}-\frac{1}{2} M a_{y} \frac{h}{t_{r}} \\
F_{z_{r r}}=\frac{1}{2} M g \frac{l_{f}}{L}+\frac{1}{2} M a_{x} \frac{h}{L}+\frac{1}{2} M a_{y} \frac{h}{t_{r}}
\end{array}\right.
$$

where:

- $g$ : gravitational acceleration,

- $a_{x}$ : vehicle's longitudinal acceleration,

- $a_{y}$ : vehicle's lateral acceleration,

- $l_{r}$ : distance between the rear axle and the vehicle's CoG,

- $L$ : vehicle's wheelbase,

- $h$ : height of vehicle's centre of gravity.

For the friction coefficient, the problem is more complex. In fact, tires are the only effectors for ground vehicles. Their behavior changes with friction. Control logic would not be as effective as in the normal situation. The changes in friction should then be predicted to reconfigure the control algorithm and act differently on tire, and on the reference in order to follow feasible targets. The difficulty is that this coefficient can only be estimated when exceeding it when using effect-based estimation methods [22]. It could be then too late to control the vehicle afterwards. Data-based techniques could provide better solutions [23]. Experimental tests showed promising results. These techniques should be further investigated. This however goes beyond the scope of this work. In this paper, we suppose that the friction coefficient can be estimated as in [24]. $\mathcal{H}_{\infty}$ synthesis has been chosen in order the generate a high-level robust controller and reduce the negative effects of this hypothesis.

The desired acceleration $\vec{v}_{\text {des }}$ in this case is:

$$
\vec{v}_{d e s}=\left[\begin{array}{c}
F_{x_{t o t}} \\
M_{z_{t o t}}
\end{array}\right]
$$

The effectiveness matrix related to control allocation precision $\mathbf{B}_{\text {eff }}$ is:

$$
\mathbf{B}_{\text {eff }}=\left[\begin{array}{cccc}
\cos \left(\delta_{f}\right) & \cos \left(\delta_{f}\right) & 1 & 1 \\
b_{2,1} & b_{2,2} & -\frac{t_{r}}{2} & \frac{t_{r}}{2}
\end{array}\right]
$$

where:

$$
\begin{aligned}
& \text { - } b_{2,1}=l_{f} \sin \left(\delta_{f}\right)-\frac{t_{r}}{2} \cos \left(\delta_{f}\right), \\
& \text { - } b_{2,2}=l_{f} \sin \left(\delta_{f}\right)+\frac{t_{r}}{2} \cos \left(\delta_{f}\right) .
\end{aligned}
$$

\section{Low-Level Control}

The low-level control corresponds to the most intern loop. Adopting a closed loop here leads to very high crossover frequencies. Moreover, the control allocation algorithm generates tire forces. Therefore, for a closed-loop low-level control, either we should be able to measure online tire forces, which is not the case in commercial vehicles, or we can use the inverse of the linear tire model with varying parameters as an interface between the two layers, and control the wheels' speed. For simplicity, and as this part goes beyond the scope of this paper, only a direct calculation is adopted:

$$
T_{b_{i j}}=-R_{i} F_{x_{i j}}
$$

Where:

- $T_{b_{i, j}}$ : brake torque at the $i-j^{4}$ wheel,

- $R_{i}$ : wheels' effective radius of axle " $i$ ".

\section{TUNABLE MOTION BEHAVIOR}

Several challenges need to be overcome regarding autonomous vehicles. Most researches focus on trajectory planning and tracking problems. However, this needs to be designed by taking into account the human in the loop. The vehicle behavior should be more similar to a human driving than a robot driving. Drivers have different profiles and different driving styles. Motions generating excitement feelings among certain people could generate in contrast fear feelings among others. The goal of this paper is to prove the ability of modern control techniques to provide different motion behaviors, and therefore different motion feelings to passengers.

Two different approaches to tune the vehicle motion behavior are exposed here. The first approach consists of modifying the motion targets to follow. The high-level control should in this case generate different generalized forces in order to amplify or reduce the vehicle response, or change its dynamics. The second approach consists of allocating differently the commands into the subsystems to generate different accelerations.

\section{A. Reference Tuning}

It should be noted that the MPC algorithm provided by LMS Imagine.Lab AMESim ${ }^{\circledR}$ can generate a steering wheel angle and a velocity profile. The MPC here can represent a driver model or a virtual driver. This paper focuses more on the vehicle motion control in general after receiving the signals generated by the MPC without differentiating between a real or virtual driver.

The yaw rate reference needs to be generated using only the steering wheel angle value and the vehicle's speed value. The bicycle model can then be used to generate the yaw rate target [25]. This simplified vehicle model represents the nominal lateral behavior of the car and is characterized by its fast computation to generate a reference, especially the static version of the model. Nevertheless, the dynamic bicycle model is used here to be able to not only generate and amplified yaw rate, but also to be able to control the transient behavior of the vehicle. The yaw rate reference is then:

$$
\dot{\psi}_{\text {ref }}=\frac{K_{\text {tun }}}{1+\tau_{\text {tun }} s} \frac{V_{x}}{L+\frac{M V_{x}^{2}}{L C_{\alpha_{f}} C_{\alpha_{r}}}\left(C_{\alpha_{r}} l_{r}-C_{\alpha_{f}} l_{f}\right)} \delta_{f}
$$

${ }^{4} i=f$ for "front" or $r$ for "rear", and $j=l$ for "left" or $r$ for "right" 
where:

- $K_{\text {tun }}$ : tuning gain,

- $\tau_{\text {tun }}$ : tuning lag,

- $C_{\alpha_{f}}$ : initial cornering stiffness of front axle when no longitudinal slip is considered,

- $C_{\alpha_{r}}$ : initial cornering stiffness of rear axle when no longitudinal slip is considered.

$K_{\text {tun }}$ and $\tau_{\text {tun }}$ can be then manually varied to see the influence on the vehicle behavior.

\section{B. Tuned Control Allocation}

It is claimed that motion sickness and discomfort are related to the vehicle's accelerations [26]. One could think of adding an additional objective to the control allocation problem to distribute differently the commands, and try to fulfill different objectives when it is possible. However, in equation (9), as we express tire forces with respect to generalized forces at the vehicle's center of gravity, we already link tire forces to accelerations. Adding another objective with almost the same effectiveness matrix leads to a rank deficiency. The optimization algorithm cannot then find a solution. This would not have been the case, if for example we want to penalize the lateral acceleration with another subsystem as the 4-Wheel Steering System. The idea is then to amplify or reduce the accelerations directly by generating amplified or reduced generalized forces. Equation (9) becomes then:

$$
\mathbf{B}_{\text {eff }} \vec{u}=\mathbf{T} \vec{v}_{\text {des }}
$$

Where $\mathbf{T}$ is a diagonal matrix with tuning parameters to amplify or reduce components of $\vec{v}_{d e s}$.

This actually prevents from several difficulties. In this way, desired accelerations are not be predefined. And there is no need to add an additional objective, which can slow down the computation. However, this can lead to control imprecision. The tuning parameters should not reach large values. Again, this may need additional experimental tests. The optimal control vector is then:

$$
\vec{u}_{\text {opt }}=\arg \min _{\vec{u}_{\text {min }} \leq \vec{u} \leq \vec{u}_{\text {max }}}\left\|\mathbf{W}_{\mathbf{c a}}\left(\mathbf{B}_{\text {eff }} \vec{u}-\mathbf{T} \vec{v}_{\text {des }}\right)\right\|^{2}
$$

The Active Set Algorithm (ASA) is then used [19]. The cost function is rewritten following the ASA formulation:

$$
\vec{u}_{\text {opt }}=\arg \min _{\vec{u}_{\text {min }} \leq \vec{u} \leq \vec{u}_{\text {max }}}\|\mathbf{A} \vec{u}-\vec{b}\|
$$

\section{CO-SIMULATION RESULTS}

As optimization-based control allocation algorithms are involved, the solver has been written in Matlab R2015a ${ }^{\circledR}$ for its strong numerical computation. Regarding LMS Imagine.Lab AMESim ${ }^{\circledR}$, it proposes a wide vehicle dynamics library for high-fidelity modeling. To take advantage from each software, both are co-simulated using Simulink ${ }^{\circledR}$ as an interface. The high, middle, and low level control logic is implemented in Simulink, while a high-fidelity car model with 15 degrees of freedom is selected in AMESim. The maneuver consists on a trajectory race tracking with highspeed values. The approved Magny-Cours area $a^{5}$ has been reproduced in 3D by AMESim developers using softwares Open Street Map and JOSM. We use then this severe maneuver to see the motion feelings generated while keeping the same trajectory.

It is important to keep the same trajectory in all simulations because we want to separate the effect of generating different trajectories for different feelings from achieving this with different accelerations (Fig.1).

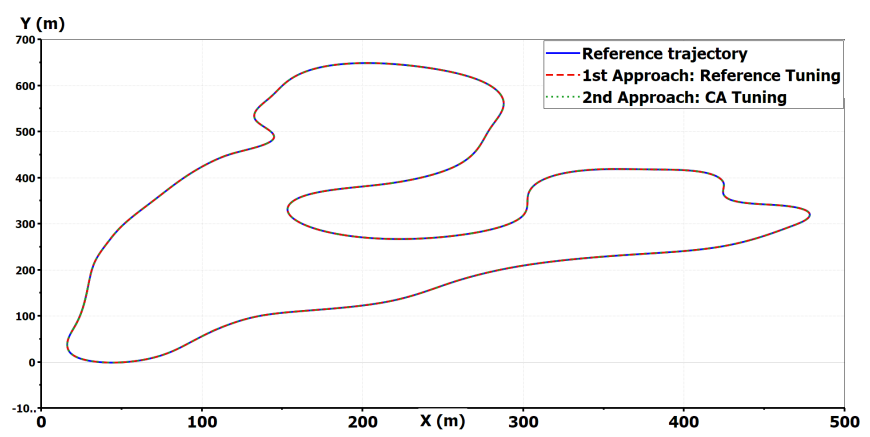

Fig. 1. Trajectories in all simulations.

\section{A. First Approach: Reference Tuning}

First, we use equation (21) to change the yaw rate reference by means of $K_{\text {tun }}$ for the response amplitude and $\tau_{\text {tun }}$ for the transient behavior. The yaw rate responses are illustrated in Fig. 2.

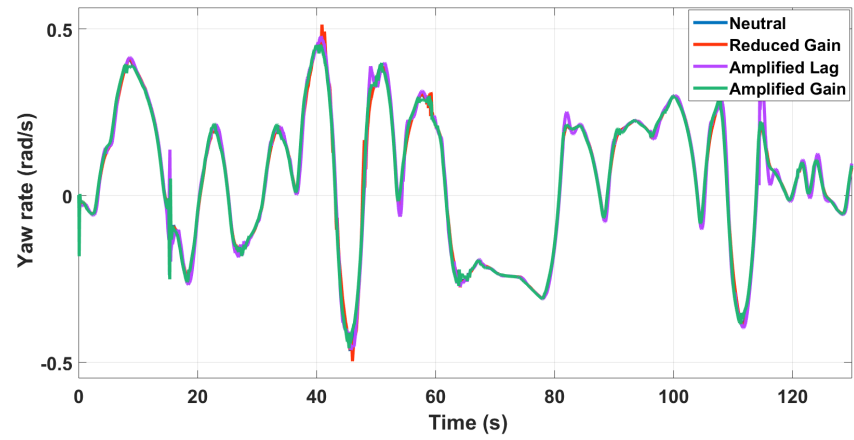

Fig. 2. Yaw rate responses in case of reference tuning.

We notice that there is not much effect on the yaw rate response. In fact, when tracking the exact same trajectory with the same velocity, the yaw rate will always be the same. So when amplifying for example $K_{t u n}$, to keep the same yaw rate, the MPC computes less front steering angle so the reference stay the same as Fig. 3 shows.

In Fig. 4 we can see that it is the difference between right and left tire forces that complements the influence of the front steering to turn the vehicle.

Therefore, this approach is not efficient regarding tuning accelerations for different feelings when there is a strict trajectory tracking along with a vehicle motion control (Fig. $5)$.

\footnotetext{
${ }^{5}$ Magny-Cours, Nevers, Niévre, Bourgogne, 58470, France.
} 


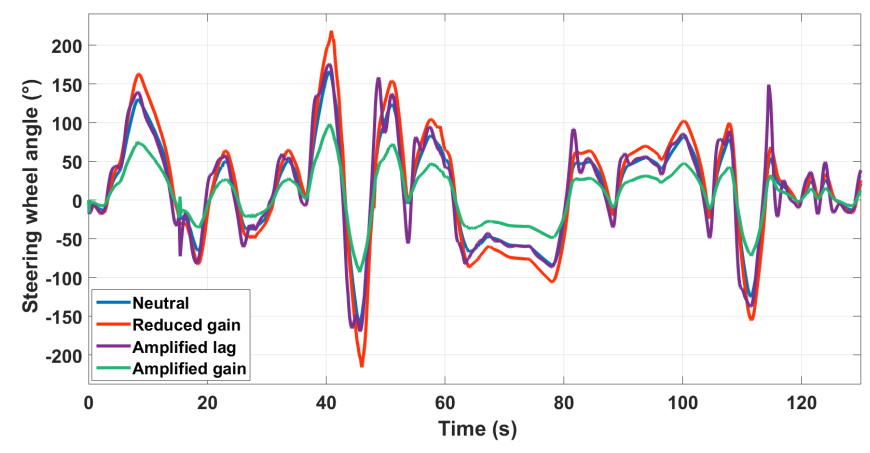

Fig. 3. Steering wheel angle in case of reference tuning.

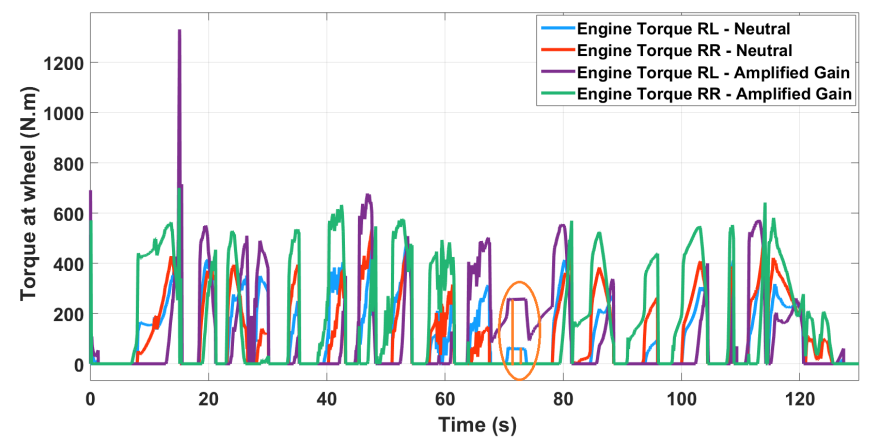

Fig. 4. Engine torques in case of reference tuning.

However, this can be relevant when there is a human driver as only few effort is needed to turn the vehicle. The torque vectoring and ESP act as assistance systems. This might also be even more beneficial for automated emergency steering for obstacle avoidance for example, as even if the driver want to take back the control, the steering wheel does not turn too much to hurt the driver's hands.

\section{B. Second Approach: Tuned Control Allocation}

Here, we penalize the forces allocation to influence directly the generated accelerations. As we can see in Fig. 6, we can obtain different lateral accelerations.

Which gives then different yaw rate responses while keeping the same trajectory (Fig. 7).

Regarding the front steering angle, this have little impact regarding the amplitude, only the transient behavior is different as Fig. 8 shows.

So to benefit from advantages of both approaches, a mixed approach is presented in the next paragraph.

\section{Mixed Approach}

Here, both the reference and the control allocation are tuned. This allows us the have different accelerations responses as Fig. 9 shows

Along with different steering wheel effort at the same time.

And all of that while keeping the same trajectory. In this way, trajectory tracking can be decoupled from motion control, and both security and comfort can be ensured. But of course, with additional chassis systems, e.g. the 4-Wheel

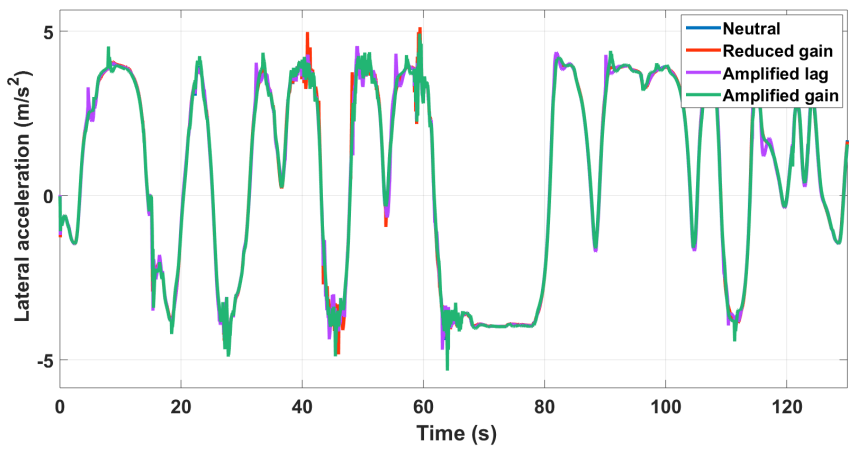

Fig. 5. Lateral acceleration in case of reference tuning.

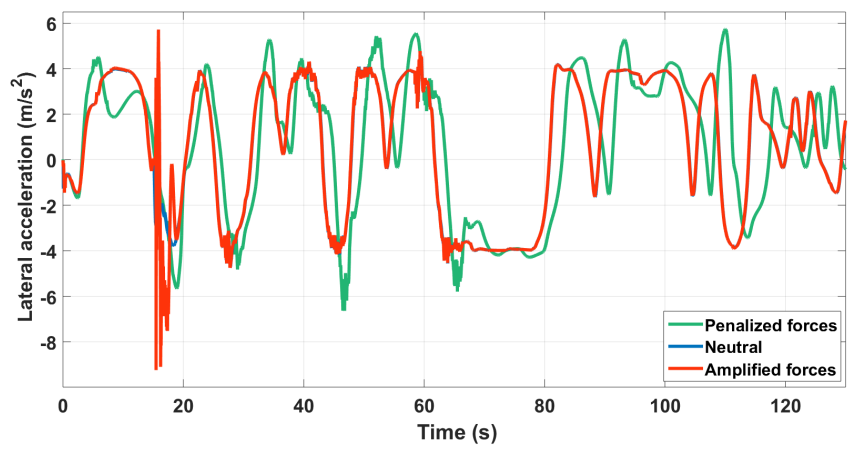

Fig. 6. Lateral acceleration in case of control allocation tuning.

Steering system, we can even allow different trajectory generation to have even more motion feelings. This corresponds to one of our future works.

\section{FUTURE CARS MOTION-RELATED CHALLENGES}

This paper aims to prove the potential of modern control technique to provide different motion feelings. This could be used to tune the vehicle behavior in order to make autonomous vehicles motions predictable and then trustful [27]. This can facilitate the acceptance of such robots/autonomous vehicles in the society and then accelerate their development. However, each driver has its own perception of what a trustful motion means. The same vehicle motion behavior can therefore generate different feelings in humans, depending on their past experience with non-autonomous vehicles. Two approaches to tune the vehicle motion behavior have been presented in this paper. The first approach consists of changing the motion reference, while the second one is based on a tuning of control allocation algorithms.

\section{A. Importance of Motion References}

In the first approach, an addition tuning gain and tuning lag have been added in order to change the response amplitude but also the transient behavior. This enables to parametrize the vehicle response to commands generated by either a human driver or a virtual driver. In fact, in case of a human drive, starting from a steering wheel angle input, the generated yaw rate by the vehicle can be amplified, 


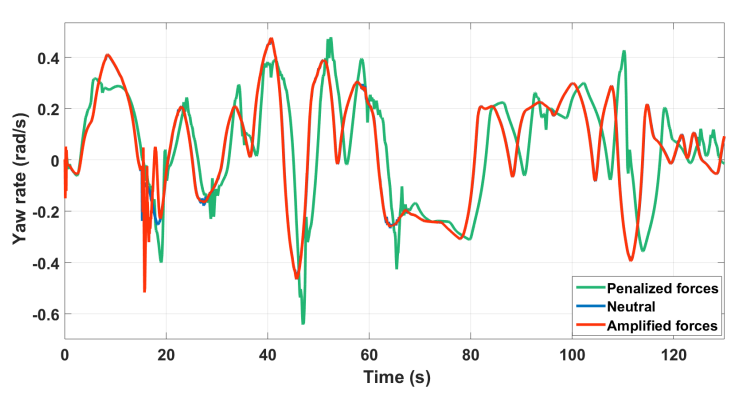

Fig. 7. Yaw rate responses in case of control allocation tuning.

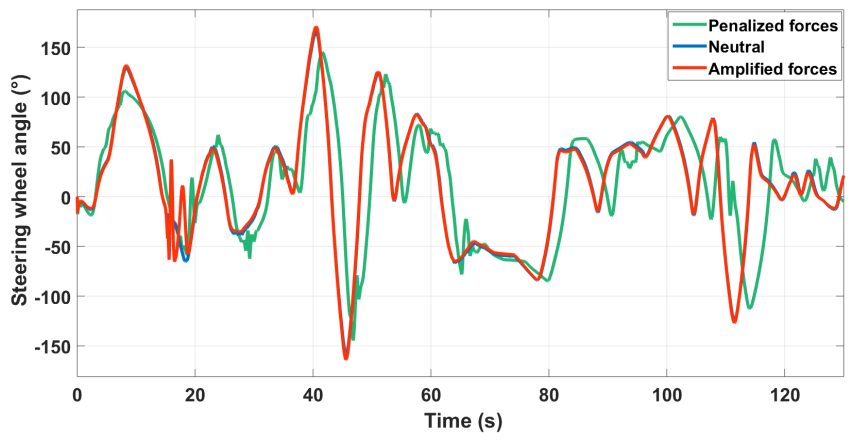

Fig. 8. Steering wheel angle in case of control allocation tuning.

reduced, delayed and so on, thanks to activation of the other subsystems. This provides different sensations to the driver itself and additional possibilities. The same logic can be applied for a virtual driver. If the MPC generates only a front steering angle to track a trajectory, the remaining systems can be used to change the dynamic responses of the vehicle. The driver can then choose among a sportive virtual driver, or a more careful virtual driver.

More complex reference models can be used to provide additional degrees of freedom. However, this additional parameters should be linked to motion feelings. Each parameter influence should be identified by experimental tests on humans. But these tests could be hard to interpret if results vary from a driver to another. A preliminary study could be necessary to classify first human drivers in order to be able to identify the influence of each parameter on generated sensations.

\section{B. Control Allocation Challenges}

Here, a simple approach has been adopted for tuning control allocation algorithms. Accelerations have been directly amplified or reduced as these variables are directly related to motion feelings. However, this approach faces the same problem as the first one. The amount of accelerations to add or reduce should be controllable and adapted to each driver. Only experimental tests involving humans could give insights about the influence of tuning control allocation objectives. Moreover, qualitative objectives as trust feelings, comfort and so one could be more sophisticated than only accelerations tuning. The control allocation problem could become a multi-objective problem with variable priorities depending

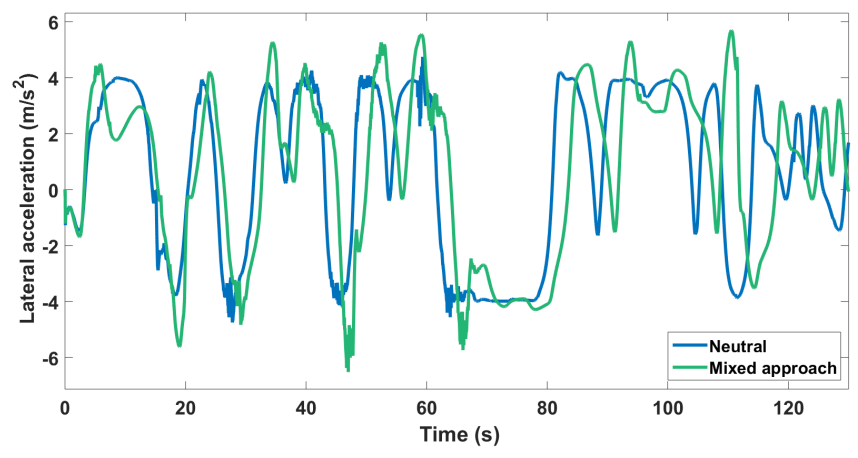

Fig. 9. Lateral acceleration in case of mixed tuning.

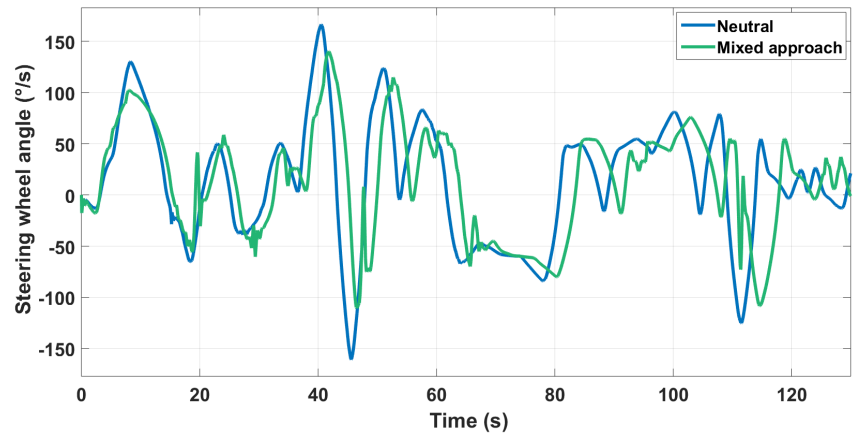

Fig. 10. Steering wheel angle in case of mixed tuning.

on the situation. In this context, authors in [28] proposed an interesting approach regarding objectives priority variance. Their approach consists on defining stabilization and trajectory tracking envelopes in the MPC algorithm where the considered states should remain. But when an obstacle has to be avoided, these envelopes can be violated in a short amount of time to ensure passenger security, then stabilize the vehicle. These adaptability should be also permitted in control allocation optimization algorithms. But again, the considered qualitative objectives should be first identified and then formalized. Moreover, adaptability could also be needed not only to take account of the environment changes, but also depending on the driving styles. As authors of [29] have reported, passengers have different expectations from autonomous driving than manual driving, as in the first case, passengers get easily bored.

Also, more chassis systems should be integrated and studied. 4-Wheels Steering (4WS) system for example could provide more comfortable and pleasant cornering maneuvers without influencing the vehicle's longitudinal speed as the brakes do [30]. This shows the lack of researches in the literature about vehicle motion control and motion feelings. More support and corporation are expected from car manufacturer for deeper investigations.

\section{CONCLUSIONS}

In this paper, two approaches for tuning vehicle behavior have been compared. While the first one consists of changing motion references dynamics, the second one consists of distributing differently the commands into subsystems by 
means of a tuning matrix to amplify or reduce accelerations. Results showed that in the first approach, the use of TV and ESP is amplified to realize maneuvers with less front steering angle, and in the second approach, accelerations have directly been tuned to enable different motion feelings. Therefore, a mixed approach have been proposed to benefit from both advantages.

This demonstrates the need of implementing this kind of modern control algorithms in future cars. The more autonomous the vehicle will become, the more authority the driver should delegate, and the more the vehicle behavior should stay predictable to prevent drivers from taking back suddenly the control.

The goal of this paper is to prove that with a multilayered architecture, control problems are separated from distribution problems. Therefore, by means of modern control techniques, additional objectives can be satisfied. For future autonomous vehicles where human acceptance is not ensured yet, this represent an opportunity to exploit more the vehicle's potential in a way to adapt its behavior to humans expectations.

The authors recognize that more evidence should be provided by means of real experiments before proposing any standards. For this reason, future works will focus on taking care of implementation issues on one hand, and study the option of generating different trajectories for more motion feelings on the other hand. We expect more collaboration from car manufacturers in order to prove our claims and participate in autonomous vehicles development.

\section{REFERENCES}

[1] M. Gerard and E.Y. Lopes, Global Chassis Control and Braking Control using Tyre Forces Measurement, TU Delft, 2011.

[2] M. Kissai, B. Monsuez and A. Tapus, Review of integrated vehicle dynamics control architectures, 2017 European Conference on Mobile Robots (ECMR), Paris, 2017, pp. 1-8.

[3] A. Soltani, Low Cost Integration of Electric Power-Assisted Steering (EPAS) with Enhanced Stability Program (ESP), PhD thesis, Cranfield University, 2014.

[4] C. O. Nwagboso, X. Ouyang, and C. Morgan, Development of neuralnetwork control of steer-by-wire system for intelligent vehicles, in Heavy Vehicle Systems, vol. 9, 2002, pp. 1-26.

[5] J.-X. Wang, N. Chen, D.-W. Pi and G.-D. Yin, Agent-based coordination framework for integrated vehicle chassis control, in Proc. Inst. Mech. Eng., D, J. Automobile Eng., vol. 223, no. 5, pp. 601-621, 2009.

[6] B. Shyrokau, D. Wang, Control allocation with dynamic weight scheduling for two-task integrated vehicle control, Proc. of the 11th International Symposium on Advanced Vehicle Control, Seoul, Korea, 2012.

[7] Y. Chen and J. Wang, Energy-efficient control allocation with applications on planar motion control of electric ground vehicles, Proceedings of the 2011 American Control Conference, San Francisco, CA, 2011, pp. 2719-2724.

[8] H. Jing, F. Jia, H. Liu and J. Sun, Multi-objective optimal control allocation for a four-wheel-independent-drive electric vehicle, 2017 36th Chinese Control Conference (CCC), Dalian, 2017, pp. 9543-9547.

[9] B. Shyrokau, D. Wang, L. Heidrich and K. Hpping, Analysis of subsystems coordination for electric vehicle during straight-line braking and brake-in-turn, 2013 IEEE Symposium on Computational Intelligence for Engineering Solutions (CIES), Singapore, 2013, pp. 61-67.

[10] S. Yim, Fault-Tolerant Yaw Moment Control with Steer and Brakeby-Wire Devices, International Journal of Automotive Technology, vol. 15, no. 3, pp. 463-468, 2014.
[11] M. Kissai, B. Monsuez, A. Tapus and D. Martinez, A new linear tire model with varying parameters, 2017 2nd IEEE International Conference on Intelligent Transportation Engineering (ICITE), Singapore, 2017, pp. 108-115.

[12] H.B. Pacejka, Tyre and Vehicle Dynamics, Second Edition, Elsevier, Butterworth-Heinemann, 2006.

[13] A. Chebly, R. Talj, A. Charara, Coupled Longitudinal and Lateral Control for an Autonomous Vehicle Dynamics Modeled Using a Robotics Formalism, IFAC-PapersOnLine, vol. 50, issue 1, 2017, pp. 12526-12532.

[14] S. Skogestad and I. Postlethwaite, Multivariable Feedback Control: Analysis and Design, 2nd Edition, JOHN WILEY \& SONS, 2001.

[15] G. Pita-Gil, Application de techniques de commande avancées dans le domaine automobile, PhD thesis, Supélec, 2011.

[16] P. Apkarian and D. Noll, "Nonsmooth H-infinity Synthesis," IEEE Transactions on Automatic Control, Vol. 51, Number 1, 2006, pp. 7186.

[17] M. Doumiati, O. Sename, L. Dugard, J. J. Martinez Molina, P. Gaspar, et al., Integrated vehicle dynamics control via coordination of active front steering and rear braking, European Journal of Control, Lavoisier, 2013, 19 (2), pp.121-143.

[18] T. A. Johansen and T. I. Fossen, Control Allocation - A survey, in Automatica, Vol. 49, Issue 5, May 2013, pp. 1087?1103.

[19] O. Harkegard, Efficient active set algorithms for solving constrained least squares problems in aircraft control allocation, Proceedings of the 41st IEEE Conference on Decision and Control, Vol. 2, 2002, pp. $1295-1300$.

[20] K.A. Bordignon, Constrained Control Allocation for Systems with Redundant Control Effectors, PhD thesis, Virginia Tech, 1996.

[21] J.J. Burken, P. Lu, Z. Wu, and C. Bahm, Two Reconfigurable FlightControl Design Methods: Robust Servomechanism and Control Allocation, Journal of Guidance, Control, and Dynamics, Vol. 24, No. 3, pp. 482-493, 2001.

[22] J. Villagra, B. d'Andréa-Novel, M. Fliess, H. Mounier, A diagnosisbased approach for tire/road forces and maximum friction estimation, Control Engineering Practice, vol. 19, issue 2, 2011, pp. 174-184.

[23] Y.-H. Liu, T. Li, Y.-Y. Yang, X.-W. Ji, J. Wu, Estimation of tireroad friction coefficient based on combined APF-IEKF and iteration algorithm, Mechanical Systems and Signal Processing, vol. 88, 2017, pp. 25-35.

[24] J. Svendenius, Tire Modeling and Friction Estimation Department of Automatic Control, Lund University, 2007.

[25] M. A. Selby, "Intelligent Vehicle Motion Control," PhD thesis, University of Leeds, Feb. 2003.

[26] F.M. Raimondi, M. Melluso, Fuzzy motion control strategy for cooperation of multiple automated vehicles with passengers comfort, In Automatica, Vol. 44, Issue 11, 2008, pp. 2804-2816.

[27] M. Hengstler, E. Enkel, S. Duelli, Applied artificial intelligence and trust - The case of autonomous vehicles and medical assistance devices, Technological Forecasting and Social Change, vol. 105, 2016, pp. 105-120.

[28] J. Funke, M. Brown, S. M. Erlien and J. C. Gerdes, Collision Avoidance and Stabilization for Autonomous Vehicles in Emergency Scenarios, in IEEE Transactions on Control Systems Technology, vol. 25, no. 4, pp. 1204-1216, July 2017.

[29] A.K. Frison, P. Wintersberger, A. Riener, and C. Schartmller, Driving Hotzenplotz: A Hybrid Interface for Vehicle Control Aiming to Maximize Pleasure in Highway Driving, In Proceedings of the 9th International Conference on Automotive User Interfaces and Interactive Vehicular Applications (AutomotiveUI '17), ACM, New York, NY, USA, pp. 236-244, 2017.

[30] H. Harada, Stability Criteria and Evaluation of Steering Maneuver in "Driver-Vehicle System", JSME international journal. Ser. C, Dynamics, control, robotics, design and manufacturing, Feb. 18, 2008. 